\title{
Prävention funktioniert - glauben wir das, oder wissen wir es?
}

Djalali, S ; Tandjung, R

\begin{abstract}
Forschung in der Hausarztmedizin betrifft nicht nur therapeutische Fragen. Eine Review untersuchte die Evidenz für Präventionsprogramme in der Schweizer Grundversorgung und deckte einen gravierenden Missstand auf.
\end{abstract}

Posted at the Zurich Open Repository and Archive, University of Zurich ZORA URL: https://doi.org/10.5167/uzh-95062

Journal Article

Published Version

Originally published at:

Djalali, S; Tandjung, R (2014). Prävention funktioniert - glauben wir das, oder wissen wir es? PrimaryCare, 14(6):97-98. 


\section{Prävention funktioniert - glauben wir das, oder wissen wir es?}

\section{Forschung in der Hausarztmedizin betrifft nicht nur therapeutische Fragen. Eine Review untersuchte die Evidenz für Präventionsprogramme in der Schweizer Grundversorgung und deckte einen gravierenden Missstand auf.}

Volksnahe Gesundheitsaufklärung und Prävention gehören zu den Kernaufgaben der Grundversorgung - aber was tatsächlich der beste Weg zu einer erfolgreichen Prävention ist, ist längst nicht klar. Um die Erfolgschancen von zukünftigen, grossangelegten, gesundheitssystemverändernden Strategien zu erhöhen, ist es deshalb notwendig, bereits bestehende Präventions- und Screeningaktivitäten objektiv zu untersuchen und hinsichtlich ihrer Erfolgsund Misserfolgsfaktoren zu analysieren. Genau das ist in dieser Review geschehen.

\section{Beliebte Ziele: Impfung und Lebensstil}

Aus allen grossen Medizindatenbanken wie Medline, Embase und der Cochrane Library wurden wissenschaftliche Arbeiten zusammengetragen, die von Präventions- und Screeningaktivitäten in der Schweizer Grundversorgung im Zeitraum von 1990-2010 berichten. Gefunden wurden 49 Studien, darunter zwölf zum Thema
Impfung und zwölf zum Thema Lebensstiländerung (Erhöhung der körperlichen Aktivität resp. Rauchstoppberatung). Diese beiden Themenkomplexe waren führend. Kleinere Anzahlen von Arbeiten bezogen sich auf die Prävention von kardiovaskulären Erkrankungen, Krebsscreening, HIV, Osteoporose und Suchterkrankungen.

\section{Ein Zeitproblem}

Als wichtigste Erfolgsfaktoren für Präventionsprogramme wurden die Motivation und die spezifische Weiterbildung der teilnehmenden Ärzte, sowie die komplikationslose Durchführbarkeit der Präventionsmassnahme im Praxisalltag genannt. Auch die Motivation des Patienten, der Grad seines medizinischen Wissens resp. seiner Aufklärung sowie die unkomplizierte Durchführung bestimmten den Erfolg der Präventionsprogramme. Als eine Art «Killerkriterium» erwies sich dagegen die Zeit. Mehrere Studien mit völlig unterschiedlichem Präventionsinhalt berichteten, dass mangelnde Zeit für die adäquate Auseinandersetzung mit dem Patienten das grösste Hindernis seien. Darüber hinaus waren mangelnde Gesprächs- und Kommunikationsfähigkeiten sowie mangelndes Hintergrundwissen über die Präventionsinhalte häufig genannte Gründe für das Nichtdurchführen von Präventionsmassnahmen.

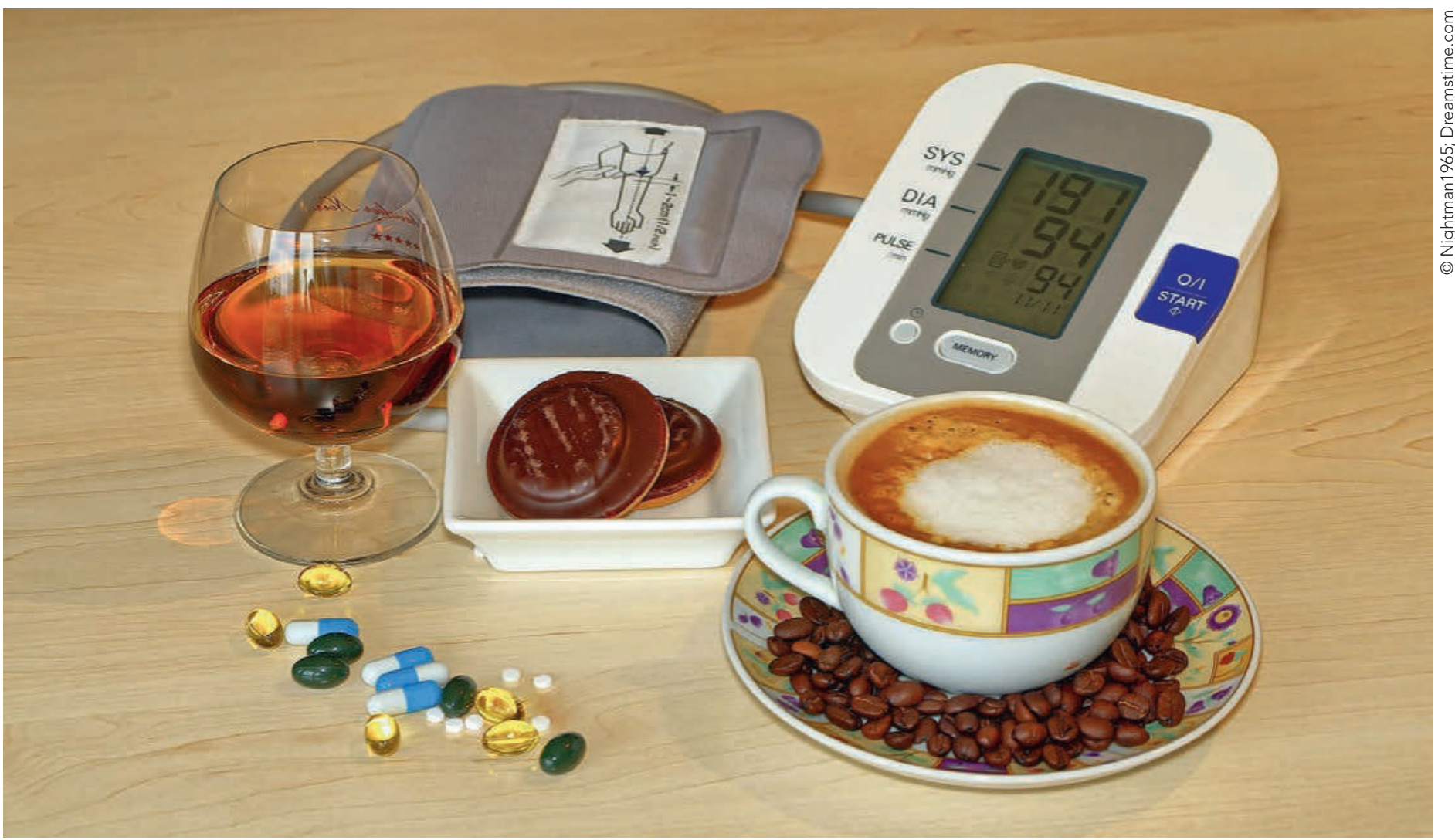

Abbildung

Macht eine Lebensstilberatung den Unterschied? Welche Form der Prävention effektiv wirkt, ist wissenschaftlich längst nicht belegt. 


\section{Mangel an Evidenz}

Das gravierendste Ergebnis, das diese Review jedoch zutage brachte, ist die Erkenntnis, dass die Evidenz auf dem Gebiet der Prävention in der Schweiz als sehr gering zu werten ist. Mehr als pauschale, tendenzielle Aussagen liessen sich aus den untersuchten Studien nicht generieren, da die wenigsten qualitativen Standards für valide wissenschaftliche Arbeiten genügten. Bei den untersuchten 49 Studien handelte es sich überwiegend $(n=45)$ um rein deskriptive Studien. Vier waren randomisiert und kontrolliert, wobei nur eine Studie methodologisch einwandfrei war. Gemessen an den international etablierten CONSORT-Kriterien (Consolidated Standards of Reporting Trials) für Studien wiesen die übrigen methodologische Schwächen aus, die die Validität der dokumentierten Ergebnisse in Frage stellen.

\section{Mehr Versorgungsforschung ist notwendig}

Diese Review zeigt einen erheblichen Mangel an guter Versorgungsforschung in der Schweiz auf. Im Grunde fehlt ein solides, valides Fundament für die Einführung von Präventionsmassnahmen. Das soll nicht das Vertrauen in die hausärztliche Tätigkeit auf dem Gebiet der Prävention untergraben - im Gegenteil, es soll dazu anhalten, die Tätigkeit beizubehalten, aber in Zukunft durch eine methodisch starke Begleitforschung zu flankieren, so dass Evidenz gewonnen werden kann. Diese kann als Grundlage für umfassende Präventionsprogramme dienen, die einen grossen Nutzen für einen möglichst grossen Anteil der Bevölkerung bringen.

\section{Literatur}

Eisner D, Zoller M, Rosemann T, Huber C, Badertscher N, Tandjung R. Screening and prevention in Swiss primary care: a systematic review. Int J of Gen Med. 2011;4:853-870.

\section{Kommentar aus der PrimaryCare-Redaktion}

Wie die Autoren im zweitletzten Satz festhalten, ist das Ergebnis der rezensierten Review-Arbeit kein Verdikt gegen Prävention und Gesundheitsförderung in der Arztpraxis. Der Befund lautet vielmehr, dass die methodische Qualität der meisten Arbeiten nicht genügt, um die Wirksamkeit der Interventionen einwandfrei zu belegen. Es ist im Bereich Prävention und Gesundheitsförderung zudem sehr zeit- und geldaufwändig, harte Endpunkte wie Morbiditäts- und Mortalitätsparameter zu messen, weil sie naturgemäss erst Jahre bis Jahrzehnte nach der Intervention eintreffen. Solche Studien sind kaum finanzierbar.

Natürlich ist eine verbesserte methodische Qualität in der hiesigen Versorgungsforschung anzustreben, und unsere aufstrebenden Hausarztinstitute arbeiten intensiv daran. Aber auch die bisherige hausärztliche Forschung, gerade im Berreich Prävention, hatte ihre Berechtigung: Forschende HausärztInnen reflektierten dadurch ihre tägliche Arbeit. Gerade beim Zeitproblem - für mich der wichtigste «Nebenbefund" aus diesem Review - sind kreative Lösungen wie Skillmix zwischen Arzt und Praxisteam gefragt. Die Botschaft lautet also nicht etwa "So nicht, Leute», sondern «Jetzt erst recht, gemeinsam mit Profis!».

Stefan Neuner-Jehle

\section{PrimaryResearch - das Fenster zur Forschung}

In dieser Artikelserie stellen wir Forschungsarbeiten aus dem Institut für Hausarztmedizin der Universität Zürich (IHAMZ) vor. Die Originalarbeiten sind entweder «open access» zugänglich oder beim jeweiligen Autor auf Anfrage erhältlich. Die Ergebnisse geben einen spannenden Einblick in die täglichen Herausforderungen, aber auch die Leistung der Hausarztmedizin. An dieser Stelle ein herzlicher Dank an alle Kolleginnen und Kollegen, die sich an den Projekten beteiligen und die hier präsentierten Ergebnisse erst ermöglicht haben!

\section{Korrespondenz:}

Dr. med. Sima Djalali

Institut für Hausarztmedizin

Universität Zürich

Pestalozzistrasse 24

8091 Zürich

sima.djalali[at]usz.ch
Universität

Zürich $^{\text {vin }}$

Institut für Hausarztmedizin 